My thanks are due to Colonel R. N. Chopra and his co-workers in the Tropical School of Medicine, Calcutta, for their assistance in the examination of the aqueous humour. I am also indebted to my House Surgeon, Dr. P. K. Biswas, for his valuable help in this research.

\title{
REFERENCES
}

1. Kirwan, E. O'G.-Arch. of Ophthal., Vol. XII, No. 1, July, 1934.

2. Chopra, R. N., Mukerjee, S. N., and Gupta, J. C.-Ind. Jl. of Med. Res., Vol. XXIII, p. 353, 1935.

\section{INTENSITY OF LIGHT IN RELATION TO THE EXAMINATION OF THE EYE*}

BY

\author{
C. E. FERree and G. RAND \\ BALTIMORE, U.S.A.
}

I. In the Correction of Errors of Refraction.

1. Myopia, hyperopia and astigmatism by the acuity method.

2. Astigmatism by special tests.

(a) With the broken circle and the illumination scale.

(b) With the radial line type of test chart.

3. The presbyopic eye for near seeing.

II. In the Standard Rating of Acuity and Its Rating for Other Purposes.

III. In the Determination of the Near Point of Vision and the Apparent Range of Accommodation.

IV. In the Study of the Visual Field with the Tangent Screen and the Perimeter.

In July, 1934, we published the description of an instrument which provides a convenient means of varying the intensity of illumination over a wide range from zero to very high without change in the colour of light or in the size, shape and location of the illuminated field. 1 This instrument was devised as an aid to the practitioner in examining the eye as well as in caring for it by prescribing the amount of light needed by the patient under examination. The use of variable illumination in the correction

* From the Research Laboratory of Physiological Optics, Baltimore, Md. 
of myopia, hyperopia and astigmatism; for the determination of the near point of vision; in the standard rating of acuity and its rating for other purposes; and in the study of the visual field with the tangent screen and perimeter have already been discussed in print and will be briefly reviewed in the following sections. Its use in the examination of the presbyopic eye and the selection of the proper strength of glasses for near work will be considered in this paper. Relating to the need for prescribing the amount of light for reading and other types of work, we have recently completed a survey on this point of more than 600 cases evenly distributed in the decade age groups from 10 to 70 years. The results of this survey will soon be published. It may be noted here, however, that in any series of tests for the prescribing of light it will be found that the major need for individual testing is for the adolescent, the sick and defective, and for the presbyopic and near-presbyopic eye. There is much less need for individual testing of normal eyes between the ages of 20 and 35 years. We have found that the greater number of these eyes prefer a medium intensity of light over a comparatively small range. In case of the presbyopic eye the greatest benefit from the test may be had for eves in the early and middle stages of presbyopia.

\section{In the Correction of Errors in Refraction}

\section{Myopia, Hyperopia and Astigmatism by the Acuity} Method, i.e., with the Conventional Letter Chart.

There are three important factors in the visibility of the image on the retina : its clearness, which is dependent on the refractive mechanism; its subjective or sensation difference from the background, which is dependent both on the physical difference in reflection between object and background and on the intensity of the illumination; and its size. It is obvious that if we wish to test the clearness of the image with the purpose of obtaining the greatest possible clearness of outline, the test should not be made in the presence of a high value of either of these other two cardinal factors. Writers and workers in this field early recognized that size of object must be reduced to a minimum if high sensitivity is wanted for the acuity method of detecting errors in refraction. No one would have recommended, for example, that large test letters be used for a decision as to which correcting glass best remedies defects in imagery. It is somewhat strange, therefore, that the other extremely important factor, namely the sensation difference from the background, should have been overlooked.

With a given physical difference from the background, i.e., a given difference in reflection factors, the apparent or sensation 
difference increases rapidly with increase in the intensity of light. This, together with the better focusing action of the refractive mechanism due to the small pupil characteristic of high intensities, gives rise to higher values of visual acuity with higher intensities of illumination. High intensities give the retina much greater power to discriminate detail in the image formed than do low intensities, therefore greater power to compensate for the error in refraction which it is the purpose of the test to disclose. Even though the image is badly blurred, the detail can be discriminated when the intensity is high. From this it follows that high intensities of light operate against the sensitivity of the test for the detection of errors in clearness of imagery by the acuity method. The test may be begun at a medium intensity, but it should be finished at a low intensity. It is our experience that except in cases where acuity is greatly reduced by causes that cannot be remedied by refractive means, intensities of $0.5 \mathrm{ft}$.-c. or less give maximum sensitivity for the final correction of myopia, hyperopia and astigmatism by this method. ${ }^{2}$

\section{Astigmatism by Special Tests.}

(a) With the use of the broken circle and the illumination scale.-Several years ago we devised a very sensitive test for astigmatism based on the use of the broken circle in connection with a variable intensity of illumination. The principle is that the opening in the circle should require the same minimum intensity of illumination for its discrimination in whatever direction it may be turned, provided the resolving or imaging power of the refracting system is equal in all meridians. If the resolving power is poorer in some one meridian than in the others, more light is required for its discrimination in that meridian. Differences in the intensity of illumination required to make the opening visible may then be used as a means of detecting differences in resolving power in the different meridians. Differences in size of opening could also be used for this purpose, but we would have to be able to produce and measure much smaller differences in size than in intensity of illumination in order to detect small differences in resolving power because of the much greater effect of size than intensity of illumination on the visibility of objects.

The illumination scale, being in comparison an amplified scale, is much more sensitive, therefore, for detecting small differences in the resolving power of the refractive system than the visual angle scale. Also changes in illumination can be more easily made in continuous series than changes in size and can be used with equal facility with any type or number of types of test object. 
The method of using the test is as follows: The amount and axis of the defect is first roughly determined by the customary less sensitive means. With the correction indicated worn by the patient, the opening of the circle is turned in succession into the two critical meridians and the amount of light required for its discrimination determined. If this is not equal, slight changes are made in the axis of the cylinder, corresponding changes being made also in the position of the opening. If this does not render equal the amount of light required to discriminate the opening in the two positions, changes are made in the strength of the cylinder and the test procedure repeated.

More recently we have shortened the procedure to one-half by using two openings in the circle with their centres displaced from

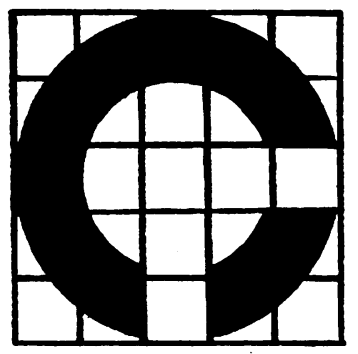

FIG. 1.

The new type of broken-circle test object drawn to the $1-5 \mathrm{~min}$. scale.

each other by 90 degrees. The use of this type of circle in no way decreases the sensitivity of the test and has, besides, the technical advantage that the test in both meridians can be made simultaneously. Simultaneous comparisons are in general always to be preferred to successive comparisons and particularly in case of astigmatism when the test is made in the absence of cycloplegia. Also, as stated above, the test with the two openings can be made in one-half the time. A representation of this circle drawn to the 1-5 minute scale is given in Fig. 1 . The test could be made with a variable illuminator such as is mentioned above, and a printed rotatable double-broken circle as test object, or less successfully with the Ferree-Rand double-broken circle chart for testing acuity and astigmatism. From the standpoint of ease and convenience of examination it can best be made with an acuity projector of the type devised by us which is provided with a double-broken circle in the form of a rotatable lantern slide and a means of varying the intensity of light. 
As illustrative of the sensitivity of the test, the following data may be cited: (a) For different amounts of astigmatism. Five observers on the average required 55 per cent. more light to detect the opening of the test object in the unfavourable than in the favourable meridian of a 0.12 dioptre astigmatism; 109 per cent. more light for a 0.25 dioptre astigmatism; and 178 per cent. more light for a 0.75 dioptre astigmatism. (b) For different amounts of astigmatism with the correcting cylinder placed off axis. Five observers on the average required 67 per cent. more light to detect the opening of the test object in the unfavourable than in the favourable meridian of a 0.25 dioptre astigmatism when the axis of the correcting cylinder was displaced 5 degrees and 96 per cent. more light when it was displaced 10 degrees; in case of a 0.75 dioptre astigmatism, they required 107 per cent. more light when the axis of the correcting cylinder was displaced 5 degrees and 160 per cent. more when it was displaced 10 degrees. In obtaining the above results artificial astigmatisms were created for $(a)$. For (b) artificial astigmatisms were created and the proper strength of correcting cylinder was placed the designated amount off axis. The experiment was conducted in this way because we wished to know the exact amount and location of the refractive defect for making the determinations. ${ }^{3}$

(b) With the radial line type of test chart.-Great sensitivity may be added to the use of charts of the radial line type by conducting the test at a very high intensity of illumination. The illuminating light should be of high intensity in order to give as much light as possible to be refracted or blurred across the black lines in the image on the retina in the meridians affected by the astigmatism. Obviously, the higher is the reflecting power of the background and the greater the intensity of the incident light, the greater will be the amount of light that is blurred across the black lines in the image and the lighter will they become. The difference in the blackness of line in the meridians affected and those not affected by the astigmatism is, it will be remembered, the crucial feature of the test.

Further, it has also been found of value in many cases of slight astigmatism to vary the illumination on the chart rapidly from high to low and back again to high in order to take advantage of the rapidly changing contrast sensitivity of the eye during change of intensity of illumination, thus accentuating the difference between the blurred grey lines and the sharply-focused black lines of the chart.

Some experiments conducted by us to show the effect of intensity of illumination on the sensitivity of the test for detecting errors in the placement of the correction may be mentioned here. Artificial astigmatisms were created by the use of 0.12 and 0.25 dioptre 
cylinders and the appropriate correcting cylinder was placed surcessively at $5,10,15,20,25,30,35$ and 40 degrees off axis. With these settings of the correcting cylinder the amounts of light were determined that were necessary for the observer just to detect the error in the placement of the correction by the difference in blackness of the lines of the chart. The results are shown in Table 1.

\section{TABLE I}

Showing the intensity of illumination of the radial line chart required to detect given amounts of error in the placement of the correction of an artificial astigmatism.

\begin{tabular}{|c|c|c|}
\hline $\begin{array}{l}\text { Strength of artificial } \\
\text { astigmatism } \\
\text { (dioptres) }\end{array}$ & $\begin{array}{l}\text { Amount correcting } \\
\text { cylinder is placed off } \\
\text { axis (degrees) }\end{array}$ & $\begin{array}{l}\text { Intensity of illumination } \\
\text { required to detect } \\
\text { error in placement of } \\
\text { correction (toot-candles) }\end{array}$ \\
\hline 0.12 & $\begin{array}{r}5 \\
10 \\
15 \\
20 \\
25 \\
30 \\
35 \\
40\end{array}$ & $\begin{array}{l}61^{\circ} 0 \\
51^{\circ} 0 \\
45^{\circ} 0 \\
40^{\circ} 0 \\
37^{\circ} 0 \\
34^{\circ} 0 \\
30^{\circ} 5 \\
28^{\circ} 0\end{array}$ \\
\hline 0.25 & $\begin{array}{r}5 \\
10 \\
15 \\
20 \\
25 \\
30 \\
35 \\
40\end{array}$ & $\begin{array}{r}50.0 \\
35.0 \\
26.5 \\
18.1 \\
11.1 \\
7.8 \\
5.3 \\
4.7\end{array}$ \\
\hline
\end{tabular}

Important factors in the sensitivity of the test are high intensity of illumination, high reflecting power of background, minimum reflecting power of the radial lines (narrow black velvet ribbon excellent for this purpose), and optimum breadth and spacing of the lines. ${ }^{4}$

\section{The Presbyopic Eye for Near Sfeing.}

There are two ways of aiding the presbyopic eye to see its object at the desired near distance, namely, a correcting glass and intensity of light. The former of these is the major and the latter a minor or auxiliary aid. However, the proper selection of either cannot be had without reference to the other. The preferred procedure is the selection of the optimum combination of intensity of light and strength of glass. 
So far as the correcting glass is concerned, it may be said that the strength of glass required at a given near distance sustains, within comparatively narrow but significant limits, an inverse relation to the intensity of light used. At high intensities the work can be discriminated with a weaker glass than at medium and low intensities. This is due, for the greater part, if not entirely, to the effect of the higher intensities of light on acuity and the visibility of the object. ${ }^{5}$ There are the following objections to the use of high intensities in selecting the correcting glass : (1) If a glass were selected at a high intensity it would have to be used at high intensities. So selected, the glasses prescribed would be unsatisfactory for work at medium and low intensities. (2) High intensities are difficult to obtain in present lighting practice with adequate protection from glare from the source of light. Also prolonged use of too high an intensity of light is apt to cause discomfort because of glare from the page or other work due to a too high brightness. (3) The glasses would have to be used in all sorts of locations and situations. High intensities are not to be found in all locations and situations, and probably never will be.

With decrease of intensity less effect of the change on the preferred strength of glass is found-little, in fact, until very low illuminations are reached. At these illuminations the tendency is to choose a stronger glass in order to secure a benefit trom magnification. This glass, however, would not, in most cases, be satisfactory at medium and high intensities without changing the distance of the work, and probably not even then because of the greater amount of convergence required, the less favourable relation between accommodation and convergence, and the undue limitation of the range of distance over which the object can be discriminated. At these intensities the additional magnification is not needed for the comfortable discrimination of the object.

Obviously, then, the practical procedure requires that in general the glass be prescribed of a strength that is most satisfactory for the range of intensities that prevails at any given time in the current practice of lighting-due exception being made for cases where the glasses are to be used only or primarily at other intensities of illumination. Assuming that this range is now from 5 to $20 \mathrm{ft}$.-c., that glass should be selected which is the most satisfactory throughout this range, the test being made a $5,10,15,20 \mathrm{ft} .-\mathrm{c}$. or at other suitable intervals.

Our experience leads us to believe that in the greater number of cases, a glass which is satisfactory for this range will also be reasonably satisfactory in case higher intensities of light are preferred because of some condition of the sensorium or for other reasons. If not, the problem becomes in such cases to find the 
best combination of strength of glass and intensity of light. With a convenient means of varying the intensity of illumination, this should not be difficult to do. The ability to control at will the important factor of intensity of light gives a certainty and finish to the test which is highly gratifying to the careful worker and is not to be had from a variation in the strength of the glass alone. We very strongly believe that some satisfactory type of variable illuminator should supplement the trial case as part of the standard equipment of the refractionist.

Although we do not consider it worth while to give here the test data for any considerable number of cases, we do, however, feel justified in citing one case which seems to be typical, as illustrative of what has been said above. A. G., male, aged 56 years, by occupation an instrument maker, preferred for reading at $30 \mathrm{ft} .-\mathrm{c} .+1.37 \mathrm{~S}$. right eye, and $+1.25 \mathrm{~S}$. left eye, in addition to his correction for distance; at 10 to $20 \mathrm{ft} .-\mathrm{c} .,+1.50 \mathrm{~S}$. right eye, and $+1.37 \mathrm{~S}$. left eye; at $5 \mathrm{ft} .-\mathrm{c}$. $+1.62 \mathrm{~S}$. both eyes; at $3 \mathrm{ft} .-\mathrm{c}$., $+1.87 \mathrm{~S}$. both eyes; and at $1.5 \mathrm{ft} .-\mathrm{c} .,+2.12 \mathrm{~S}$. both eyes. His preferred intensity of illumination for reading was 11 to $12 \mathrm{ft}$.-c.; his toleration for intensity for comfortable reading ranged from 5 to $16 \mathrm{ft}$.-c. His preferred combination of strength of glass and intensity for reading was, then, $+1.50 \mathrm{~S}$. right eye, $+1.37 \mathrm{~S}$. left eye, and 11 to $12 \mathrm{ft}$.-c. of illumination. This combination had all the desired requirements for reading, namely, the intensity of light preferred, a strength of glass that was entirely comfortable, and a range of distance over which the object could be seen with satisfactory clearness greater than for any other combination that was comfortable. The favourable range of distance here was due both to lesser strength of glass and to the very satisfactory visibility given by the comparatively high intensity of light. The combination of $+1.37 \mathrm{~S}$. right eye, and $+1.25 \mathrm{~S}$. left eye, and $30 \mathrm{ft}$.-c. of illumination was not preferred because this intensity was glaring and higher than his limit of toleration for comfortable reading. The glass chosen for this illumination was too weak for intensities of $20 \mathrm{ft}$.-c. and less. On the other hand, the glass chosen for $1.5 \mathrm{ft}$.-c. was too strong to be comfortable at 3 or more ft.-c. This glass was chosen only because a benefit of magnification was needed to overcome the low visibility due to the small amount of light. The glasses chosen for 3 and $5 \mathrm{ft} .-\mathrm{c}$. were, however, comfortable at higher intensities up to his upper limit of toleration for intensity of light for reading, but were not preferred at these intensities because they were stronger than was needed and, therefore, limited unduly the range of distance over which the object could be comfortably discriminated. While he had facilities in his own shop for $10 \mathrm{ft}$.-c. and more, his work was on dark material, and often required him to go from room to room to examine and 
repair apparatus where that much illumination was not provided. In such a case we would not feel justified in prescribing the glasses that were preferred at higher than 3 , or at most, $5 \mathrm{ft}$.-c.

We would again urge that due attention be given to both factors, strength of glass and intensity of light, in making a prescription for either. This indicates clearly that the prescribing of glasses and the prescribing of light should be done by one who is competent to give both factors due consideration. Often too strong glasses are prescribed because too low an intensity of light has been used in making the examination ; and conversely, frequently too high an intensity of light is advised or required because the person is wearing too weak a glass, in which case he may be compelled for the sake of adequate visibility to work above his threshold of glare.

This discussion, it will be understood, has no adverse bearing on any ideal or just desire to raise the general level of intensity in lighting practice. In fact, the procedure recommended is the authorized way to bring about an increase in the amount of light used by that part of our population most in need of it, namely, the middle-aged and old-an increase that will have permanence and stability because it is based on the sound foundation of a physiological requirement. Obviously, the safe and sure aid to advancement in the solution of the problem of intensity is through some authentic test procedure or other competent means of advisement supplemented by convenient and satisfactory ways and devices of adapting intensity to the variable needs. Any plan or course of action not based on a reasonably thorough understanding of the ocular situation is apt to prove detrimental and reactionary.

The raising of the level of intensity to any considerable extent will come only with great labour and great patience of endeavour. The prescribing of glasses cannot wait for the desired progress in that direction. We are dealing here with a practical situation in the present. When such progress comes, it can be taken into account in the test procedure; that is, the test will be made at higher intensities of light. At these intensities a weaker glass will be preferred for near work, which will have the very desirable feature of a greater range of distance over which the glasses will provide clear seeing. Also the glasses will not need to be changed so frequently as presbyopia advances. Both of these are very important, particularly the range of distance over which the object can be seen clearly. It may be added, however, that in connection with the raising of the level of intensity, full consideration must be given to the threshold of glare. This varies a great deal in different individuals, but in the same individual does not seem to be materially affected by strength of glass over the range that is apt to be prescribed. Also it is considerably lower for 
artificial light than for either daylight or artificial light properly corrected for colour. Further, we are inclined to believe, both from items of our own experience and from the experience of others, that it is lower even for sunlight than for daylight.

\section{In the Standard Rating of Acuity and its Rating for other Purposes}

For a suitable intensity of illumination of the test charts used in making standard ratings of visual acuity, it might be suggested that an attempt be made to approximate the standard of lighting practice. However, standards of practice in commercial lighting change greatly from time to time and, moreover, it is difficult at any one time to determine just what the standard is. Again, the current aim in commercial lighting seems to be by the use of high intensities to give all eyes good vision; that is, to eliminate as far as possible individual differences. This is hardly suitable for a scale the purpose of which is to rate all eyes fairly as to their powers of vision. Further, the higher intensities do not sufficiently rule out those disqualified by age in cases where the standard rating of acuity is to be used as the basis of selection for special fitness. For this purpose it would seem better to select a medium intensity, for example, $10 \mathrm{ft}$.c. For the sake of simplicity of specification and of equipment required, the intensity could, without sacrifice of fitness for the purpose, be made the same as is now commonly accepted for perimetry and the study of the visual field, namely, 7 ft.-c.

In the rating of eyes as to fitness for vocations, the test should be made as nearly as possible at the illumination usually employed in the vocation in question. The study of even a small number of cases shows that eyes are not given the same relative rating as to acuity at different intensities of illumination. For example, experience has shown in the U.S. Navy that only 25 to 30 per cent. of the men accepted for the service on the basis of the conventional acuity test made at the higher illuminations are able to qualify for the look-out work at night on the bridge of battleships. Further, in a test of 61 observers made by us, all under 28 years of age and rating $6 / 4$ acuity by the conventional test with $5 \mathrm{ft}$.-c. of light on the test chart, 13 per cent. rated below $6 / 6$ at $0.55 \mathrm{ft}$.-c., and 33 per cent. below $6 / 6$ at $0.2 \mathrm{ft}$.-c. The acuity of the remainder was $6 / 6$ or better at these illuminations. It is quite obvious that any attempt to rate eyes for vocational purposes at only one, or even one order of intensity of illumination is based on a lack of knowledge of the differential effect for different eyes of intensity of illumination on the power of the eye to see clearly. 
Perhaps nowhere in the examination of the eye is there shown a greater need for a ready means of varying the intensity of the illumination and of measuring what is produced than in the rating of acuity in its various applications. At present there is great confusion as to the ratings made in relation to special fitness and the comparative ratings made of the same person at different times and in different places. ${ }^{6}$

\section{In the Determination of the Near Point of Vision and}

\section{the Apparent Range of Accommodation}

There is a considerable effect of increase of intensity of light on the distance of the near point of vision and therefore on the apparent range of accommodation. The effect is present at all ages, but is much greater for middle-aged and old than for young eyes and for presbyopic than for non-presbyopic eyes.

For example, in a group of 12 observers without presbyopia, ranging in age from 20 to 41 years, we have found that by increasing the intensity of light from 1 to $5 \mathrm{ft}$.-c., the distance of the near point was decreased for the different individuals $1.8-26 \mathrm{~mm}$., or 2-26 per cent. ; for an increase of from 1 to $25 \mathrm{ft} .-\mathrm{c}$., the distance of the near point was decreased $3-45 \mathrm{~mm}$., or $3-28$ per cent. In a group of 14 presbyopic observers, ranging in age from 40 to 53 years and in strength of correction for near vision from 1 to 2 dioptres, an increase of intensity from 1 to $5 \mathrm{ft}$.-c. decreased the distance of the near point $90-540 \mathrm{~mm}$., or $18-51$ per cent.; while an increase of intensity from 1 to $25 \mathrm{ft}$.-c. decreased the distance of the near point $170-690 \mathrm{~mm}$., or $32-73$ per cent.

The increase in apparent range of accommodation for these observers expressed in dioptres was as follows: For the group without presbyopia, an increase in intensity from 1 to $5 \mathrm{ft}$.-c. increased this range 0.18-1.19 dioptres, or 2-19 per cent. for the different observers; and an increase from 1 to $25 \mathrm{ft}$.-c. increased it 0.27-2.38 dioptres, or 3-39 per cent. For the group of presbyopic observers, an increase of intensity from 1 to $5 \mathrm{ft}$.-c. increased the apparent range of accommodation 0.33-1.3 dioptres, or 22-105 per cent.; and an increase of intensity from 1 to $25 \mathrm{ft}$.-c. increased this range $0.61-4.32$ dioptres, or $48-477$ per cent.

In addition to showing the very great benefit of intensity of light to the near vision of older and particularly of presbyopic eyes, these results demonstrate the need for the adoption of some fixed value of intensity for all standard determinations of the near point and range of accommodation. There is no reason why this intensity should not be the same as was recommended for the 
standard rating of visual acuity. When used in conjunction with the prescribing of glasses, however, the values of the near point should also be established and recorded for the intensity of light for which the reading glasses are prescribed. ${ }^{7}$

\section{In the Study of the Visual Field with the}

\section{Tangent Screen and Perimeter}

Studies of the visual field are very important in all pathological conditions of the sensorium. Within a 30-degree distance of the centre of the field these studies can be made with either the tangent screen or the perimeter. Outside this distance the perimeter should be used. The variable illuminator mentioned earlier in the paper is very useful for studies with the tangent screen. For the FerreeRand perimeter we have provided a special form of illuminating device having the same type of intensity control as is used in our variable illuminator, which is attached to the instrument.

The following uses and advantages of variable illumination in connection with studies of the visual field may be briefly mentioned :

(1) As the initial step in the routine procedure for determining the limits of the visual field and blind spot, the study should always be made at the intensity of illumination which is at present accepted as standard, $7 \mathrm{ft}$. - . The results may then be compared with the norms for these limits which have been established at that intensity.

(2) In testing for incipient pathological conditions, sensitivity can be added to the study by reducing the visibility of the stimuli employed. Decrease of intensity of the illumination of test object and background when the test objects are either coloured or neutral, is one of the three methods of decreasing visibility feasible to employ for this purpose. The other two methods are decrease in the size of coloured or colourless test objects, and decrease in difference in reflection factor between test object and background when the test objects are not coloured. The amount of increase in sensitivity that can be secured in these three ways is limited only by the threshold of visibility. By employing singly or in combination any of these methods of decreasing the visibility of the test objects, faint or incipient scotoma, regional cuts in the visual field, or significant irregularities of outline may be picked up which cannot be detected under the conditions of visibility noted in (1). The early detection of such defects in the field is, of course, extremely important in diagnosis. In the less incipient cases where a defect can be detected by the conditions noted in 
(1), the more sensitive procedure is also of value in showing the further spread of the disturbance. ${ }^{8}$

(3) In cases where the pathological condition is well advanced, particularly when only a small segment of the field is sensitive to the test objects ordinarily used, it is desirable to be able to increase the visibility of the test objects in suitably graded steps in order to investigate the regions of the retina where the sensitivity is so reduced that the standard test objects fail to arouse response. In many cases this type of study proves to be very valuable in both diagnosis and prognosis. It is of especial interest in showing the differences in shape of field as outlined by stimuli at different levels of visibility. The conditions for obtaining test objects of higher visibility are, of course, the reverse of those listed above for reducing visibility. That is, high intensities of illumination, large sizes of test object and large differences in reflection factor between test object and background, accomplish this purpose within the limits of increase of visibility by these means.

(4) In all other cases a much more comprehensive picture of the extent and shape of the area of disturbance is obtained by using test objects of lower and higher visibility than would be had by using the standard test objects and conditions alone. This also gives valuable information pertaining to the course the pathological disturbance has taken and is taking.

The most effective way of varying the visibility of test objects for the analysis and study of the visual field is by making the gross changes by increasing or decreasing the size of the test objects or the difference in reflection factor between test object and background, and the finer changes by varying the intensity of illumination of the test objects.

(5) In many types of pathological condition a very valuable contribution to the study may be made by determining the threshold of response to coloured and neutral stimuli in various significant parts of the field, central and peripheral. The sensitivity gradient can be determined, if desired, in any direction in any part of the field or in as many meridians as may be wanted from centre to periphery. With such possibilities there is practically no limit to the thoroughness with which the study of the field or any part of it can be made. With either the variable illuminator and tangent screen combination, or with the Ferree-Rand perimeter equipped with the special illuminating device with intensity control mentioned above, this very important work has now been made not only possible but convenient.

In case of non-pathological eyes the following effects of the visibility of the stimulus on the extent and shape of the fields for form and colour may be noted. 
(1) With different visibilities of stimulus the size of the form field may be made to change from full to comparatively small. With change in size there is also change in shape. This is due to the unequal sensitivity gradients in the different meridians. ${ }^{9}$

(2) With sufficiently high visibility of stimulus (high intensity spectrum light, background matched as nearly as possible in brightness to stimulus), the fields for red, blue and yellow may be made co-extensive with the form field. With stimuli of lesser visibility the fields for colour may be made to decrease in size, ranging from large to very small. The decrease in size is attended with still greater changes in shape than for the form field. This is due to the greater differences in the sensitivity gradients for colour than for form from meridian to meridian..$^{10}$

(3) With a suitable selection of ratios of visibility of the stimuli, the comparative sizes of the fields for blue, red, green and yellow may be made to vary from greater to less in any sequence desired. With stimuli of approximately equal energy of the order of visibility that would cause the limits to fall in the mid-periphery of the field, the limits for blue, red, green and yellow interlace. With stimuli of equal energy of a higher order of visibility, the limits for blue, red and yellow interlace; for most observers the limits for green are narrower. This interlacing of limits is due to change in the ratio of sensitivity to the different colours from meridian to meridian. The order of ranking of size of field : blue, red, green, usually obtained with pigment stimuli in medical perimetry, is due to the comparative visibilities of the stimuli employed and does not represent the comparative sensitivity of the retina to these colours. ${ }^{10}$

From the above facts it is obvious that all comparative studies of the extent and shape of the fields for form and colour must be made under controlled conditions of visibility and that for diagnosis critical values for the limits are needed under some set conditions accepted as standard. ${ }^{11}$

Some mechanical means of varying the intensity of illumination is the most satisfactory for the purposes discussed above. In our variable illuminator we have used a shutter the contiguous vanes of which rotate in opposite directions in closing in order to avoid any change in the placement of light, and a diffusing means sufficiently adequate to eliminate all shadows and to give an evenly distributed, well diffused illumination. Under no consideration should a rheostat be used for this purpose because of the radical distortion in colour value produced, particularly at the lower intensities of illumination. With filter means, it is difficult to get adequate durability, neutrality of colour and fineness of steps of change over a sufficiently wide range. 


\section{REFERENCES}

1. Ferree, C. E., Rand, G.-Lamp for determination and measurement of the preferred intensity of light for reading and for other work. Arch. of Ophthal., Vol. XII, pp. 45-59, 1934.

2. The effect of increase of intensity of illumination on acuity and the question of the intensity of illumination of test charts. Amer. Jl. of Ophthal., Vol. VI, p. 672-675, 1923.

The testing of visual acuity. I. Factors in the sensitive use of the test for the detection of errors in refraction. Ibid., Vol. XVII, pp. 29-36, 1934.

3. - Visual acuity at low illumination and the use of the illumination scale for the detection of small errors in refraction. Ibid., Vol. III, pp. 408-417, 1920.

Sensitivity of the illumination scale for determining exact amount and placement of correction for astigmatism. Ibid., Vol. IV, pp. 22-27, 1921.

The testing of visual acuity. II. The comparative merits of test objects and a new type of broken circle as test object. Ibid., Vol. XVII, pp. 610-618, 1934.

The testing of visual acuity. III. Types of test field and a projection apparatus. Ibid., Vol. XVII, pp. 1147-1150, 1934.

4. Intensity of illumination and other factors influencing the sensitivity of the radial line test for astigmatism. Ibid., Vol. XII, pp. 809-814, 1929.

5. - The effect of variations in intensity of illumination on functions of importance to the working eye. Trans. Ill. Eng. Soc., Vol. XV, pp. 769-792, 1920.

Intensity of light and speed of vision studied with. special reference to industrial situations, Part I. Trans. Ill. Eng. Soc., Vol. XXII, pp. 79-110, 1927 ; Part II, Ibid., Vol. XXIII, pp. 507-542, 1928.

Ferree, C. E., Rand, G., and Lewis, E. F.-Age as an important factor in the amount of light needed by the eye. Arch. of Ophthal., Vol. XIII, pp. 212-226, 1935.

6. Ferree, C. E., Rand, G.-The effect of increase of intensity on acuity and the question of the intensity of illumination of test charts. Amer. Jl. of Ophthal., Vol. VI, pp. 672-675, 1923.

7. - Intensity of light in relation to the near point and the apparent range of accommodation. Ibid., Vol. XVIII, pp. 307-318, 1935.

8. Methods for increasing the diagnostic sensitivity of perimetry and scotometry with the form field stimulus. Ibid., Vol. XIII, pp. 118$120,1930$.

Ferree, C. E., Rand, G., and Sloan, L. L.-Sensitive methods for the detection of Bjerrum and other scotomas. Arch. of Ophthal., Vol. V, pp. 224-260, 1931.

Roenne's nasal step as studied with stimuli of different visibilities. Ibid., Vol. VI, pp 877-900, 1931.

9. Ferree, C. E., Rand, G.-The effect of relation to background on the size and shape of the form field for stimuli of different sizes. Amer. Jl. of Ophthal., Vol. XIV, pp. 1018-1029, 1931.

10. The absolute limits of colour sensitivity and the effect of intensity of light on the apparent limits. Psychol. Rev., Vol. XXVII, pp. 1-23, 1920.

The effect of intensity of stimulus on the size and shape of the colour fields and their order of ranking as to breadth. Amer. Jl. of Ophthal., Vol. VI, pp. 453-460, 1923.

The effect of variations of the intensity of the illumination of the perimeter arm on the determination of the colour fields. Psychol. Rev., Vol. XXIX, pp. 457-473, 1922.

The effect of size of stimulus on size and shape of colour fields. Amer. Jl. of. Ophthal., Vol. X, pp. 399-411, 1927.

Chromatic thresholds of sensation from centre to periphery of the retina and their bearing on colour theory. Psychol. Rev., Vol. XXVI, pp. $16-41,150-163,1919$. 
Wentworth, H. W.-A quantitative study of achromatic and chromatic sensitivity from centre to periphery of the retina. Psychol. Monog., Vol. XL, 189 pp., 1930.

11. Ferree, C. E., Rand, G., and Monroe, M. M.-Studies in perimetry. I. Preliminary work on a diagnostic scale for the form field. Amer. Jl. of Ophthal., Vol. IX, pp. 95-104, 1926.

Studies in perimetry. II. Preliminary work on a diagnostic scale for the colour fields. Ibid., Vol. XII. pp. 269-285, 1929.

Studies in perimetry. IV. Preliminary work on a diagnostic scale for the form field with a 0.17 degree stimulus. Ibid., Vol. XIII, pp. 859-867, 1930.

Diagnostic scales for the 1 degree and 0.17 degree form field stimuli for the eight principal meridional quadrants taken separately. Arch. of Ophthal., Vol. VI, pp. 518-534, 1931.

Critical values for the limits of the colour fields in the eight principal meridional quadrants taken separately. Amer. Jl. of Ophthal., Vol. XVI, pp. 577-589, 1933.

\title{
THE RELATIONSHIP BETWEEN CONJUNCTIVITIS AND TRACHOMA*
}

\author{
BY \\ A. F. MacCallan, C.B.E. \\ PRESIDENT OF THE INTERNATIONAL ORGANIZATION \\ AGAINST TRACHOMA
}

I HAVE been requested to address this General Assembly of the International Association for the Prevention of Blindness and of the International Organization against Trachoma on "The Relationship between Conjunctivitis and Trachoma."

The term conjunctivitis is applied to any form of inflammatory change affecting the conjunctiva, while trachoma is a variety of conjunctivitis, the full name of which is conjunctivitis trachomatosa.

In order to recognise trachoma a definition is required, and I give the following formula :

Trachoma is a specific contagious disease of the conjunctiva in man. It is chronic in nature. It is characterized by a subepithelial infiltration of the conjunctiva by a cellular exudate, which spreads to the cornea and to the tarsus. It. is followed by cicatricial changes in the affected tissues.

That the disease is specific and contagious has been proved over and over again by the experimental or accidental inoculation of a healthy human conjunctiva from that of a trachomatous individual. The disease is chronic, such acute manifestations as occur being due, in the majority of cases, to superimposed

\footnotetext{
* Presidential Address at the session of the League against Trachoma, held in Paris, May 9-15, 1936.
} 